\title{
O CRESCIMENTO ECONÔMICO SOFRE INFLUÊNCIA DA INOVAÇÃO E DA CULTURA NACIONAL?
} ECONOMIC GROWTH SOUNDS THE INFLUENCE OF NATIONAL INNOVATION AND CULTURE?

Adriana Kroenke ${ }^{1}$

Paula Carolina Ferretti ${ }^{1}$ Jandir Tambosi Junior ${ }^{1}$

Recebido em: 26/09/ 2018 Aceito em: 11/12/2018

akroenke@furb.br
Resumo: Neste artigo, verificou-se a influência da inovação e cultura nacional no PIB per capita de 66 países. Utilizou-se dados secundários disponibilizados no Banco Mundial, para o PIB per capita, do Índice de Inovação Global, para a inovação nacional e do sítio eletrônico Hofstede Insight, para a cultura nacional. Analisou-se os dados por meio de dois modelos de regressão linear múltipla. Os resultados indicam que as variáveis inovação e eficiência de inovação influenciam o PIB per capita, da mesma forma, quatro variáveis da cultura nacional, sendo distância do poder, individualismo, orientação a longo prazo e indulgência, relacionamse ao PIB per capita dos países estudados. Apenas a masculinidade e aversão à incerteza, variáveis de cultura, não influenciam o PIB per capita.

Palavras-chave: Inovação Nacional. Cultura Nacional. Produto Interno Bruto per capita.

Abstract: In this article, the influence of national innovation and culture in the GDP per capita of 66 countries was verified. Secondary data available at the World Bank for GDP per capita, the Global Innovation Index for national innovation and the Hofstede Insight website for national culture were used. Data were analyzed by means of two multiple linear regression models. The results indicate that the innovation and innovation efficiency variables influence per capita GDP, in the same way, four variables of the national culture, being distance of power, individualism, long-term orientation and indulgence, are related to the per capita GDP of the countries studied. Only masculinity and aversion to uncertainty, culture variables, do not influence GDP per capita.

Keywords: National Innovation. National Culture. Gross Domestic Product per capita.

\footnotetext{
${ }^{1}$ Universidade Regional de Blumenau - FURB - Blumenau - Santa Catarina - Brasil.
} 


\section{INTRODUÇÃO}

O crescimento econômico nacional é um dos principais focos da política econômica dos países, sendo um recurso influenciado por vários aspectos culturais ou sociais (SHARIPOV, 2016). Alguns indicadores foram estudados e possuem ligação com o crescimento econômico, relacionandose com mudanças tecnológicas (ROMER, 1990), exportações (ESFAHANI, 1991), dívidas nacionais (PATTILLO; POIRSON; RICCI, 2004) e concorrência entre países (BANYA; BIEKPE, 2017). Há a necessidade de revisar os conceitos básicos da teoria econômica a medida que a inovação e aprendizagem são colocadas como elementos de mensuração, enfatizando a participação do governo na construção de tecnologia e desenvolvimento econômico (LUNDVALL, 2016).

As mudanças culturais são interligadas ao comportamento social e acabam por implicar no desempenho da inovação de um país, refletindo em decisões econômicas (KOSTIS; KAFKA; PETRAKIS, 2018). As dimensões da cultura nacional possuem influência nas taxas de adoção de inovações pelas empresas (WAARTS; EVERDINGEN; VAN, 2005). Além disso, sugere-se que as diferenças culturais entre os países acabam por afetar a adoção de inovação e que o efeito da inovação depende do contexto cultural em que está inserido (EVERDINGEN; VAN; WAARTS, 2003; RAUCH et al., 2013).

As políticas de inovação empregadas nas nações, refletem na competitividade econômica tanto para países em desenvolvimento, quanto para países emergentes (AGUIRRE-BASTOS; WEBER, 2017). Sociedades altamente inovadoras têm características culturais de individualismo, com pouca distância de poder e aceitação de incerteza (SHANE, 1993; SHANE; VENKATARAMAN; MACMILLAN, 1995). Nesta perspectiva, o presente estudo tem por objetivo analisar a influência da inovação e da cultura nacional no PIB per capita de países. Diante do exposto, elaborou-se a seguinte pergunta de pesquisa: Qual a influência das variáveis de inovação e cultura nacional no PIB per capita de um conjunto de países?

Ademais, este estudo apresenta justificativas teóricas e práticas. Teoricamente, em termos de estudos que relacionem a cultura e a inovação nacional, encontrou-se diversas pesquisas, contudo, a relação destas variáveis comparadas ao PIB per capita, não retornaram elevado número de pesquisas, espera-se então, expandir os conhecimentos científicos acerca deste tema. Empiricamente, os resultados obtidos sugerem que a inovação nacional e algumas dimensões de cultura nacional podem elevar o crescimento econômico, tendo como perspectiva o PIB per capita. Com base nisso, os tomadores de decisões nacionais, poderão propagar o crescimento econômico de seus países por meio de incentivos à inovação e análise da cultura local.

\section{FUNDAMENTAÇÃO TEÓRICA}

Apresenta-se nesta seção os principais tópicos que contribuirão para o entendimento das variáveis utilizadas neste estudo. Assim, destacam-se a inovação nacional, a cultura nacional e o Produto Interno Bruto (PIB) per capita.

Estudos do CEPE [ISSN 1982-6729]. Santa Cruz do Sul, n. 48, p. 146-161, jul./dez. 2014. https://online.unisc.br/seer/index.php/cepe/index 


\subsection{Inovação Nacional}

A interpretação de sistemas nacionais de inovação foi introduzida no final da década de 1980 por Freeman (1987) e Dosi et al. (1988), após alguns anos, Lundvall (1992), Nelson (1993) e Edquist (1997) continuaram a desenvolver o termo (CHEN; GUAN, 2012). Ao final dos anos 1980 e início dos anos 1990, a literatura da inovação passou a fundamentar-se na política, direcionando-se a todos os agentes que influenciavam a inovação (FAGERBERG; SAPPRASERT, 2011). Dentre os envolvidos no processo de inovação nacional, destacam-se a indústria e seu ambiente, o governo e a universidade. O principal objetivo deste sistema é a inovação (GODIN, 2009).

Nenhuma definição foi adotada com unicidade para o sistema nacional de inovação, contudo, em seu conceito geral, é definido como um conjunto de instituições que produzem inovação e que permitem intermédio do governo para a criação de políticas públicas que incentivem esta atividade (CHEN; GUAN, 2012). No âmbito nacional, pode-se encontrar as políticas de inovação, em que, o objetivo é a competividade econômica tanto em países emergentes como em países em desenvolvimento (AGUIRRE-BASTOS; WEBER, 2017).

Há duas vertentes que podem se diferenciar ao definir sistemas nacionais de inovação. A primeira refere-se ao contexto geográfico, na qual espera-se a condução de uma região com razoável relação com os processos de inovação, sendo locais, regionais, nacionais e supranacionais. A segunda, relaciona-se aos sistemas de inovação delimitados setorialmente, ou seja, um conjunto específico de tecnologia ou áreas de produto, incluindo somente uma parcela do sistema internacional, nacional ou regional (JOHNSON; EDQUIST; LUNDVALL, 2003).

Para definir e identificar um sistema nacional de inovação de forma ampla, alguns aspectos devem ser considerados (LUNDVALL, 2016). Na abordagem ampla de sistemas nacionais de inovação, as instituições que promovem inovação em segmentos específicos fazem parte de um sistema socioeconômico extenso, englobam questões culturais, políticas e econômicas, além de auxiliarem na projeção de atividades inovadoras (FREEMAN, 2002).

A inovação não ocorre isoladamente, isto é, necessita-se de uma integração de diferentes fatores que permitam relacioná-la com o crescimento econômico. Isto requer que se vá além da infraestrutura científica. Inclui-se neste processo, instituições e organizações que participem na construção de competências no mercado de trabalho, na educação e na vida profissional (LUNDVALL, 2016). A inovação e o desenvolvimento financeiro são impulsionadores fundamentais para o crescimento econômico per capita à longo prazo (PRADHAN; ARVIN; BAHMANI, 2018).

A tecnologia é colocada em evidência quando se trata de inovação nacional. As capacidades tecnológicas das organizações são fonte de competitividade, sendo possível identificar as diferenças e semelhanças existentes entre as nações, podendo explicar a variação no desempenho econômico nacional (NELSON, 1992) e diagnosticar a direção na qual um país conduz-se pela velocidade e aprendizado tecnológico (PATEL; PAVITT, 1994). Pode-se verificar pelas organizações internacionais mudanças tecnológicas e assim obter aplicação de medidas políticas tecnológicas com o intuito de melhorar a inovação nacional (BALZAT; HANUSCH, 2004). 
O avanço tecnológico possui um papel fundamental no crescimento econômico e este é justamente a ideia principal dos sistemas nacionais de inovação (NELSON; NELSON, 2002). Considera-se, portanto, que o sistema nacional de inovação é um índice que colabora com a identificação do crescimento econômico, possibilitando não apenas as indicações sobre planejamento de inovações e acompanhamento da competitividade para os responsáveis políticos, como também sendo fonte de estudos para pesquisadores no campo da inovação e economia (LU; KWEH; HUANG, 2014).

Os governos nacionais preocupam-se de forma específica com a eficiência da inovação. Este índice auxilia na compreensão do conceito de produtividade, onde verifica-se a quantidade de insumos de inovação na produção de mais inovação no país, ou ainda, se menos insumos de inovação são utilizados para a mesma produção de inovação. Assim, é possível compreender o potencial máximo de um país em entradas e saídas de inovação (CHEN; GUAN, 2012).

O conceito de sistema nacional de inovação, pode então, resumir-se em um dado que contribui para a verificar os diferentes meios de inovar em aspectos essenciais para a construção de inovação em países em desenvolvimento (JOHNSON; EDQUIST; LUNDVALL, 2003). A inovação é um instrumento estratégico para o crescimento de um país. Reconhecer e interpretar a inovação nacional permite aos países emergentes buscarem soluções ao crescimento econômico, inclusive aos seus futuros empreendedores e inovadores (GII, 2017). Neste sentido, pretendeu-se nesta pesquisa, direcionar informações que contribuirão para os países estudados no aspecto econômico interligando cultura e inovação.

\subsection{Cultura Nacional}

O termo cultura geralmente é enquadrado em nações, grupos étnicos ou regionais, podendo ser acrescentado em aspectos organizacionais, familiares e profissionais (HOFSTEDE, 1980). A pesquisa realizada por Hofstede, tem por objetivo formular uma terminologia empírica e diagnosticar uma coleta de dados extensa sobre diversas culturas (KWOK; TADESSE, 2006).

Conforme mostra-se no quadro 2 , no aprofundamento do conceito de cultura nacional de Hofstede, destacam-se seis dimensões que permitem o entendimento de como é estruturado estes indicadores. A primeira dimensão trata da distância de poder, sendo voltado à desigualdade de uma nação (HOFSTEDE, 2011). Em estudos anteriores, Zhao, Li e Rauch (2012) e Ostapenko (2015), analisaram que a distância do poder tem impacto no desenvolvimento do empreendedorismo nacional, sendo influenciado pelo ambiente sociocultural, a distância do poder explica diferenças na quantidade de atividades empreendedoras entre as sociedades.

A segunda dimensão é a aversão à incerteza, indicando o quanto uma sociedade é preparada para lidar positivamente ou negativamente com situações não previstas com antecedência, ou seja, situações desconhecidas (HOFSTEDE, 2011). O papel da cultura nacional é extenso e implica também nos sistemas financeiros de um país, conforme verificado com Kwok e Tadesse (2006), países com maior incerteza são mais propensos a terem um sistema baseado em bancos. 
A terceira dimensão refere-se ao individualismo, neste ponto, surge também o coletivismo. No coletivo, os países prezam pela integração entre grupos, do contrário, acredita-se que cada indivíduo deve cuidar de seus problemas sem intervir no coletivo (HOFSTEDE, 2011). Couto e Vieira (2004), concluíram que a cultura nacional influencia a pesquisa e desenvolvimento de um país. Quanto maior o individualismo, maior é o nível de atividades de pesquisa e desenvolvimento.

$\mathrm{Na}$ quarta dimensão destaca-se a masculinidade versus a feminilidade. Esta dimensão é relativa as questões de distribuição de valores entre os gêneros (HOFSTEDE, 2011). A cultura nacional afeta a atividade empresarial e o comércio internacional, principalmente nas dimensões de masculinidade e feminilidade (KRISTJÁNSDÓTTIR et al., 2017).

$\mathrm{Na}$ quinta dimensão encontra-se a orientação a longo prazo. Em sociedades que adotam a cultura da orientação a curto prazo, as tradições são consideradas sagradas, do contrário, quando a longo prazo, as tradições são moldadas conforme as mudanças ocorrem (HOFSTEDE, 2011). Ao compreender os efeitos que a mudança cultural causa na inovação, os envolvidos nas decisões políticas de um país poderão preparar-se de forma mais eficiente para disseminar condições necessárias à cultura da inovação local (KOSTIS; KAFK; PETRAKIS, 2018).

A sexta e última dimensão, denomina-se indulgência. Em um país que os índices de indulgência são altos, o lazer é colocado como um meio importante na vida das pessoas, quando a restrição é tida como mais alta, diminui-se a importância do lazer (HOFSTEDE, 2011). A influência da cultura nacional no comportamento dos indivíduos é colocada em foco quando pretende-se analisar possíveis falhas nas decisões, e voltar-se para a inteligência das nações (ANGELIS, 2016). A cultura nacional ainda é vista como influenciadora na geração de renda e emprego, indiferente de que campo a cultura esteja vinculada, seja lucrativo ou não lucrativo, público ou privado, com raízes no patrimônio ou na indústria criativa (BANDARIN; HOSAGRAHAR; ALBERNAZ, 2011).

\subsection{Produto Interno Bruto per capita}

O Produto Interno Bruto é determinado pela soma do consumo de bens e serviços durante um ano, estimados pelos preços de mercado, que equivale a renda primária do país, sendo assim, o PIB é definido por três enfoques: produção, demanda e renda (IBGE, 2016). Para calcular o valor do PIB per capita utiliza-se o valor do PIB dividido pelo número de habitantes no território nacional (IBGE, 2014). O PIB é um indicador utilizado para realização de comparações internacionais entre economias e pode ser útil para verificar se a quantidade de bens e serviços produzidos per capita na economia nacional, estão crescendo ou diminuindo (AGUADO; MARTINEZ, 2012).

Dentre os determinantes mais relevantes que afetam o crescimento econômico, encontra-se os fatores endógenos e exógenos. Os fatores exógenos caracterizam-se pela geografia, instituições, tendências demográficas, fatores socioculturais e fatores políticos. Nos fatores endógenos, estão o capital físico, capital humano, pesquisa e desenvolvimento, políticas econômicas, condições macroeconômicas e abertura ao comércio (SHARIPOV, 2016). O PIB per capita tem sido utilizado na literatura para compreender as condições econômicas (MONTAÑÉS; OLMOS; REYES, 2018) além 
de servir como indicador para a qualidade de vida em diferentes contextos nacionais (ANDREANO; LAURETI; POSTIGLIONE, 2013).

Os fatores de oferta e demográficos ao longo prazo representam um papel significativo no crescimento do PIB per capita, economias que possuem melhor desempenho no mercado de trabalho estão interligadas com maior produtividade, logo, suas taxas de crescimento do PIB per capita são maiores (MARATTIN; SALOTTI, 2011). O investimento estrangeiro direito (IED) e o capital humano foram considerados fatores importantes no crescimento do PIB (SU; LIU, 2016). Da mesma forma, o nível de desenvolvimento regional e o nível de capital humano mensurados pelo PIB per capita possuem correlação (LASKOWSKA; DAŃSKA-BORSIAK, 2016).

\section{METODOLOGIA}

A presente pesquisa classifica-se como descritiva por apresentar planos estruturados, descrevendo características de uma amostra por meio de uma pergunta de pesquisa (HAIR JR. et al., 2005), tendo como objetivo analisar a influência da inovação e cultura nacional no PIB per capita de 66 países. A abordagem caracteriza-se como quantitativa pois é uma das formas pela qual pode-se testar teorias objetivas a fim de verificar a relação existente entre as variáveis. Com isso, as variáveis podem ser medidas por meio de instrumentos, possibilitando que os dados sejam testados estatisticamente (CRESWELL, 2010). No caso desta pesquisa, utiliza-se métodos quantitativos, mais precisamente regressão linear múltipla para analisar influências.

Tratando-se dos procedimentos adotados, o estudo classifica-se como documental, visto que, para realizar a pesquisa utilizou-se dados do site de Hofstede Insight (2017), que apresentam dimensões da cultura nacional. O banco de dados do Índice de Inovação Global (2017), utilizado para compor as informações de inovação nacional e os dados do PIB per capita, retirados do Banco Mundial (2018). Por se tratar de uma coleta documental, os dados dispostos foram possíveis pela interação entre as informações disponíveis em ambos os bancos de dados.

No quadro 1 apresenta-se os indicadores que descrevem as variáveis independentes da inovação nacional. Estes dados foram retirados do relatório do Índice de Inovação Global, do ano de 2017, isso dá-se ao fato de ser o relatório mais recente. O Índice de Inovação Global possui um rico banco de dados de métricas detalhadas para economias. No ano de 2017 abrangeu 127 países, que representam $92,5 \%$ da população mundial e $97,6 \%$ do PIB global. Além disso, é considerado referência em inovação nos últimos 10 anos (GII, 2017).

Quadro 1 - Indicadores da inovação nacional

\begin{tabular}{|c|l|}
\hline Indicadores & \multicolumn{1}{c|}{ Descrição do indicador } \\
\hline Score Inovação & $\begin{array}{l}\text { Score de Inovação é uma métrica de avaliação para avaliar as inovações das } \\
\text { nações. }\end{array}$ \\
\hline $\begin{array}{c}\text { Índice de } \\
\text { Eficiência da } \\
\text { Inovação }\end{array}$ & $\begin{array}{l}\text { O Îndice de Eficiência em Inovação representa a quantidade de inovação } \\
\text { gerada por um país com base em seus insumos. }\end{array}$ \\
\hline
\end{tabular}

Estudos do CEPE [ISSN 1982-6729]. Santa Cruz do Sul, n. 48, p. 146-161, jul./dez. 2014. https://online.unisc.br/seer/index.php/cepe/index 
No Quadro 2 descreve-se as dimensões que compõem as variáveis independentes da cultura nacional. Os dados de 2017 foram coletados no site Hofstede Insight, utilizou-se esta fonte de dados pelo acesso livre e por disponibilizar as dimensões necessárias ao estudo. Este site é pioneiro ao incentivo de pesquisas acadêmicas voltadas para a área de negócios e tem como intuito dar continuidade ao trabalho do Prof. Geert Hofstede, que é considerado um dos principais representantes de estudos e pesquisas interculturais, por meio de diversas atividades acadêmicas e culturais que vem realizando em diferentes países (HOFSTEDE INSIGHT, 2017).

Quadro 2 - Dimensões da cultura nacional

\begin{tabular}{|c|c|}
\hline Dimensões & Descrição das dimensões \\
\hline Distância de Poder & $\begin{array}{l}\text { Trata-se de um indicador que apresenta o grau de desigualdade entre os } \\
\text { níveis hierárquicos organizacionais, um número baixo para este indicador } \\
\text { demonstra que a sociedade é mais igualitária enquanto números elevados } \\
\text { demonstram uma sociedade com maior nível hierárquico e desigual. Algumas } \\
\text { características desta dimensão são: } \\
\text { - Pessoas que ocupam posições mais elevadas na estrutura } \\
\text { organizacional deliberar sobre as decisões sem consultar as pessoas } \\
\text { em posições inferiores; } \\
\text { Pessoas ocupantes em posições superiores devem evitar a interação } \\
\text { - Pocial com pessoas em posições inferiores; } \\
\text { Pessoas em posições mais baixas na hierarquia devem obedecer às } \\
\text { decisões tomadas por pessoas em níveis hierárquicos superiores. }\end{array}$ \\
\hline Indiv & $\begin{array}{l}\text { Trata-se de um indicador das nações em torno de características de } \\
\text { individualismo e coletivismo. Sociedades com baixo grau de individualismo } \\
\text { tende a privilegiar o coletivo. Algumas características desta dimensão são: } \\
\text { - As lideranças estimulam seus liderados a trabalharem em grupo; } \\
\text { - Tomam decisões em grupo; } \\
\text { - Resultados de líderes levam em consideração a qualidade da } \\
\text { participação do grupo. }\end{array}$ \\
\hline Mas & $\begin{array}{l}\text { Refere-se a um indicador que apresenta a separação de funções por sexo: } \\
\text { masculinos e femininos. Sociedade com baixos índices para este indicador } \\
\text { demonstram que o poder e sucesso são distribuídos entre homens e } \\
\text { mulheres. Algumas características desta dimensão são: } \\
\text { - As atividades são distribuídas por sexo, ou seja, determinadas } \\
\text { atividades são realizadas pelo sexo feminino e outra pelo sexo } \\
\text { masculino e a alternância destes papeis podem ser maus visto pela } \\
\text { sociedade, refletindo assim em um nível alto para este indicador. } \\
\text { - O poder é distribuído independente do gênero. }\end{array}$ \\
\hline Incerteza & $\begin{array}{l}\text { Trata-se de um indicador que demonstra o grau de ansiedade da nação em } \\
\text { torno de situações duvidosa ou incertas. Um alto índice demonstra uma } \\
\text { sociedade bastante regrada, de leis duras e bastante formalismo nos } \\
\text { negócios. As características desta dimensão são: } \\
\text { - Formalidade nos negócios; } \\
\text { - Estrutura organizacional forte; } \\
\text { - Evitam riscos. }\end{array}$ \\
\hline Orientação a longo & Refere-se ao indicador de busca de resultados de longo prazo. Nações com \\
\hline
\end{tabular}




\begin{tabular}{|c|l|}
\hline prazo & $\begin{array}{l}\text { alto índice demonstra uma sociedade mais tradicional, que respeitam as } \\
\text { tradições e a cultura de seus ancestrais, sendo assim menos criativas. } \\
\text { Algumas características desta dimensão são: }\end{array}$ \\
- $\quad$ Pessoas com maior idade possuem mais respeito que os jovens; \\
- $\quad$ Base familiar como base da sociedade. \\
\hline Indulgência & $\begin{array}{l}\text { Trata-se de uma dimensão que demonstra como a sociedade trata seus } \\
\text { impulsos e desejos. Nações com alto grau neste indicador demonstra que a } \\
\text { sociedade busca a felicidade e o prazer de seus impulsos e desejos. } \\
\text { Algumas características desta dimensão são: } \\
\text { - Busca da felicidade e alegria; } \\
\text { Pouca ansiedade com prazos. }\end{array}$ \\
\hline
\end{tabular}

Fonte: Elaborado pelos autores com base em Hofstede (1980).

Para a variável dependente PIB per capita, os dados foram retirados do Banco Mundial do ano de 2018, por tratar dos dados mais recentes e acessíveis. O Banco Mundial possibilita a transparência e compartilhamento de seus dados com o intuito de permitir que acadêmicos e outros interessados possam ampliar o conhecimento de questões globais que impactam o desenvolvimento dos países, tendo em vista que, é uma instituição que visa o conhecimento (BANCO MUNDIAL, 2018).

Dando-se sequência ao estudo, os dados foram preparados para análise de dois modelos de regressão linear múltipla. Para Maroco (2003), a regressão linear múltipla caracteriza-se por um conjunto de procedimentos estatísticos que permitem verificar o valor de uma ou mais variáveis dependentes em relação ao conjunto de variáveis independentes. Tendo este conceito, utilizou-se o modelo de regressão linear múltipla imposto por Maroco (2003):

$$
\mathrm{Y}=\beta_{o}+\beta_{1} X_{1}+\beta_{2} X_{2}+\ldots+\beta_{\mathrm{n}} X_{\mathrm{n}}+\varepsilon
$$

Para analisar a influência das variáveis de inovação no PIB per capita utilizou-se o modelo 1 com duas variáveis independentes:

$$
\mathrm{Y}=\beta_{\circ}+\beta_{1}\left(\mathrm{SI}+\beta_{2}(S E I)+\varepsilon(1)\right. \text { onde: }
$$

SI: Score de inovação

SEI: Score de eficiência da inovação

De similar modo, para analisar a influência das variáveis de cultura nacional no PIB per capita, utilizou-se o modelo 2 com seis variáveis independentes:

$$
\mathrm{Y}=\beta_{0}+\beta_{1}(D P)+B_{2}(I)+B_{3}(M)+B_{4}(A V I N)+B_{5}(O L P)+B_{6}(I N D)+\varepsilon(2) \text { onde: }
$$

DP: Distância de poder

Estudos do CEPE [ISSN 1982-6729]. Santa Cruz do Sul, n. 48, p. 146-161, jul./dez. 2014. https://online.unisc.br/seer/index.php/cepe/index 
I: Individualismo

M: Masculinidade

AVIN: Aversão à Incerteza

OLP: Orientação a longo prazo

IND: Indulgência

Nesta concepção, procurou-se após a realização da análise da regressão linear múltipla, verificar quais variáveis independentes podem influenciar a variável dependente. Na sequência, apresenta-se a análise dos resultados desta investigação.

\section{ANÁLISE E DISCUSSÃO DOS RESULTADOS}

Para verificar a possibilidade de utilizar a análise de regressão múltipla neste estudo, aplicouse o teste ANOVA. Para este teste os parâmetros de regressão em conjunto devem ser iguais a zero, caracterizando-se assim uma relação estatisticamente significativa. Como observado nas tabelas $1 \mathrm{e}$ 2, os dois modelos obtiveram ANOVA de 0,000, logo, possibilitou-se seguir com a análise.

$\mathrm{Na}$ Tabela 1 verifica-se os resultados obtidos na regressão linear múltipla utilizando as variáveis independentes de inovação nacional. De acordo com Maroco (2003), o $\mathrm{R}^{2}$ ou coeficiente de determinação, é popularmente utilizado como uma das medidas da qualidade de ajustamento. Para o $\mathrm{R}^{2}$ ser considerado adequado deve-se atentar a subjetividade de cada estudo, porém, para ciências sociais os valores de $\mathrm{R}^{2}$ devem ser acima de 0,5 para considerar-se dados aceitáveis.

Desta forma, nota-se uma correlação de $79,9 \%$ entre a variável dependente PIB per capita com as variáveis independentes de inovação nacional que se apresentaram significativas, atestando um bom ajustamento do modelo aos dados. Para Hair Jr. et al. (2005), uma variável só pode ser considerada significativa se estiver com significância abaixo de 0,05 . Portanto, conforme a tabela 1 , as duas variáveis de inovação nacional apresentaram-se significativas.

Tabela 1 - Regressão linear múltipla para variáveis da inovação nacional

\begin{tabular}{lcccc}
\hline \multicolumn{1}{c}{ Variáveis } & B não padronizado & B padronizado & Sig & VIF \\
\hline Constante & 30622,529 & & 0,000 & \\
\hline Score Inovação & 1803,173 & 1,036 & 0,000 & 1,835 \\
\hline $\begin{array}{l}\text { Score Eficiência da } \\
\text { Inovação }\end{array}$ & 37232,836 & $-0,236$ & 0,003 & 1,835 \\
\hline $\mathbf{R}$ & 0,894 & & & \\
\hline $\mathbf{R}^{2}$ & 0,799 & & & \\
\hline Durbin-Watson & 1,618 & & & \\
\hline ANOVA & 0,000 & & & \\
\hline Fonte: Dados da Pesquisa. & & & & \\
\hline
\end{tabular}

Fonte: Dados da Pesquisa. 
Na Tabela 2 apresenta-se o segundo modelo de regressão linear múltipla com as variáveis independentes de cultura nacional. Observa-se uma correlação de 67,3\% entre a variável dependente PIB per capita com as variáveis independentes distância de poder, individualismo, masculinidade, aversão à incertezas, orientação a longo prazo e indulgência. Sendo assim, para as variáveis independentes de cultura nacional, o ajustamento do modelo aos dados também pode ser considerado bom. As variáveis de cultura nacional, distância de poder, individualismo, orientação a longo prazo e indulgência apresentaram uma significância abaixo de 0,05, permitindo verificar uma relação entre quatro das seis dimensões.

Realizou-se também o Teste de Durbin Watson para avaliar o pressuposto de independência dos resíduos. Segundo Field (2009), o teste de Durbin Watson avalia a satisfação da hipótese de independência dos erros ou resíduos. Em ambas as regressões os resultados do teste de Durbin Watson apresentaram valores aceitáveis. Sendo assim, observa-se que não existe auto correlação serial entre os resíduos.

Interpretou-se ainda nas tabelas 1 e 2 o fator de influência da variância (VIF) que mede o efeito das variáveis independentes sobre o coeficiente de regressão. Segundo Fávero et al (2009), uma estatística VIF igual ou maior que 5, indica a existência de multicolinearidade entre as variáveis explicativas do modelo proposto. Para ambos os modelos apresentados observa-se que não há multicolinearidade, ou seja, nenhuma variável apresentou VIF igual ou maior que 5 .

Tabela 2 - Regressão linear múltipla para variáveis da cultura nacional

\begin{tabular}{lcccc}
\hline \multicolumn{1}{c}{ Variáveis } & B não padronizado & B padronizado & Sig & VIF \\
\hline Constante & 19890,083 & & 0,128 & \\
\hline Distância de Poder & $-368,762$ & $-0,390$ & 0,002 & 2,575 \\
\hline Individualismo & 266,81 & 0,298 & 0,015 & 2,548 \\
\hline Masculinidade & $-32,981$ & $-0,033$ & 0,671 & 1,100 \\
\hline Incertezas & $-93,914$ & $-0,097$ & 0,222 & 1,117 \\
\hline $\begin{array}{l}\text { Orientação a Longo } \\
\text { Prazo }\end{array}$ & 205,104 & 0,227 & 0,013 & 1,427 \\
\hline Indulgência & 224,713 & & 0,009 & 1,465 \\
\hline $\mathbf{R}$ & 0,821 & 0,245 & & \\
\hline $\mathbf{R}^{2}$ & 0,673 & & & \\
\hline Durbin-Watson & 2,513 & & & \\
\hline ANOVA & 0,000 & & & \\
\hline
\end{tabular}

Fonte: Dados da Pesquisa. 
De acordo com os resultados obtidos neste estudo, a inovação nacional é condizente ao crescimento econômico, sendo este medido pelo PIB per capita dos países estudados. Acredita-se que as dimensões que influenciam ao PIB per capita nesta análise, referem-se ao que sugeriu Andreano, Laureti e Postiglione (2013), de que o PIB per capita pode ser utilizado para verificar a qualidade de vida de uma nação. Consequentemente, a inovação envolvendo aspectos abrangentes como mercado de trabalho, educação e vida profissional (LUNDVALL, 2016), tem a competência de elevar índices de qualidade de vida, e estes estão associados ao crescimento econômico. Os resultados para as variáveis de inovação, vêm ao encontro do proposto por Pradhan, Arvin e Bahmani (2018), de que a inovação impacta o crescimento econômico per capita à longo prazo.

Em relação aos resultados para as variáveis de cultura nacional, uma possível explicação para que a distância do poder tenha sido significativa, pode ser considerada pelo estudo de Zhao, Li e Rauch (2012) e Ostapenko (2015), de que a dimensão de distância do poder é relacionada com o nível de empreendedorismo nacional, pois possui influências no ambiente sociocultural. Sendo assim, propõe-se que a distância do poder, voltada para níveis hierárquicos e de desigualdade, podem elevar normas e impor níveis de empreendedorismo em uma nação, logo, quanto maior for a distância do poder, menor é a chance de um país abrir novos empreendimentos e expandir economicamente, corroborando aos estudos de Shane (1993) e Shane, Venkataraman e Macmillan (1995).

A dimensão de individualismo sendo influenciadora ao PIB per capita pode ser relacionado ao fato mencionado por Couto e Vieira (2004), de que quanto maior o individualismo de um país, maior é seu nível de pesquisa e desenvolvimento. Desta forma, considera-se que o individualismo ao intensificar pesquisa e desenvolvimento em âmbito nacional, é também responsável por abranger o crescimento e o desenvolvimento econômico.

A orientação a longo prazo é tida nesta análise, como variável interveniente ao PIB per capita, uma relação provável para este resultado é condizente com o conceito utilizado por Hofstede e Minkov (2010), uma cultura que adota a orientação a longo prazo, preza pela aprendizagem, honestidade, adaptabilidade, responsabilidade e autodisciplina, sendo assim, acredita-se que estes fatores acabam por impactar no crescimento econômico e na erradicação da pobreza de determinado país.

A última dimensão que foi significativa em relação ao PIB per capita nesta análise, refere-se à indulgência. Isso pode ser explicado por meio do estudo realizado por Bandarin, Hosagrahar e Albernaz (2011), em que, a geração de renda e emprego é influenciada pela cultura nacional, em indústrias criativas. Nota-se que indicadores altos para indulgência tem reflexos na busca pela felicidade dos indivíduos da sociedade. Sendo assim, presume-se que para obter renda e emprego, melhorando índices de crescimento de um país, o capital humano deve ser considerado e verificado como foco cultural, isto vai ao encontro do que propôs Sharipov (2016), o capital humano afeta o crescimento econômico.

Como variáveis de cultura nacional que não se caracterizaram neste estudo como influenciadoras ao PIB per capita, têm-se a masculinidade e a aversão à incerteza. A aversão à incerteza é uma dimensão que identifica o nível de ansiedade dos indivíduos de uma nação, para lhe 
dar com situações duvidosas e a masculinidade é relacionada a distinção de funções entre homens e mulheres.

Julga-se que então que, estas variáveis quando presentes em uma nação não refletem ao crescimento econômico. Seguindo os descritos de Freeman (2002), a inovação requer atividades inovadoras, sendo assim, geralmente é reflexo de novas escolhas, proporcionando modificações constantes, logo, necessita de pessoas que aceitem incertezas, mudanças, erros, isso pode estar associado ao que propôs Shane (1993) e Shane, Venkataraman e Macmillan (1995), países que aceitam a incerteza são altamente inovadores, por tanto, o contrário também pode ocorrer.

Em relação a masculinidade, países com desigualdades de gênero impedem que pessoas do sexo feminino, muitas vezes qualificadas, assumam cargos de liderança, além disso, sugere-se para este resultado que, a má distribuição do poder e do sucesso pode acarretar decisões isoladas, gerando consequências ao desenvolvimento e automaticamente, ao crescimento do país em questão. Desta forma, a aversão à incerteza e a masculinidade acabam por impedir oportunidades de crescimento em um país.

\section{CONSIDERAÇÕES FINAIS}

Em geral, os resultados apontam que os indicadores de inovação nacional possuem relação com o PIB per capita. As variáveis independentes de score de inovação e score de eficiência de inovação se apresentaram significativamente influenciadoras do PIB per capita. Ao analisar as variáveis independentes de cultura nacional, pôde-se perceber que as dimensões de cultura nacional que influenciam o PIB per capita são distância de poder, individualismo, orientação a longo prazo e indulgência. As dimensões de masculinidade e aversão à incerteza não se mostraram significativas em relação ao PIB per capita.

Sendo assim, acredita-se que o crescimento econômico de um país pode ser influenciado pela inovação e cultura nacional, tendo em vista que, os resultados obtidos neste estudo comprovam estatisticamente esta relação. Uma possível explicação para estes resultados seria o fato de que quanto mais a inovação nacional é impulsionada, mais o país tende a se desenvolver e tornar-se uma nação competitiva globalmente, ou seja, isto levaria ao seu crescimento econômico. Da mesma forma, as características da cultura de um país podem direcioná-lo a contextos de maior ou menor desenvolvimento econômico.

As variáveis de masculinidade e de aversão à incerteza não evidenciam nesta pesquisa, influenciarem o PIB per capita. Este resultado pode estar associado ao fato de que países com alto grau de incerteza resistem à mudanças e são altamente ligados à regras e burocracias, acredita-se então, que países com alta aversão à incerteza acabam por não influenciar ao PIB per capita, tendo em vista que, a alta burocratização e a falta de incentivo a mudanças atreladas à inovação podem prejudicar o crescimento econômico nacional. Da mesma forma, acredita-se que em países com alta masculinidade, a sociedade sofre por desigualdades de gênero, este fato pode causar impactos negativos no crescimento econômico pois acaba por não disponibilizar oportunidades de desenvolvimento profissional para todos os cidadãos de forma igualitária e justa. 
É válido destacar que os países diferem em suas características, ou seja, países de alta renda diferenciam-se dos países com baixa renda nos tipos de atividades econômicas. Nesta concepção, sugere-se como estudos futuros, a análise das diferenças existentes entre os países em relação a inovação e cultura nacional que podem prejudicar seu crescimento econômico. Embora o período em anos não seja longo, foi possível encontrar resultados significativos. Sugere-se então que, pesquisas futuras analisem os dados com base num período mais longo.

Em termos acadêmicos, espera-se ter contribuído com a expansão de pesquisas vinculadas ao crescimento econômico por meio do PIB per capita relacionando a cultura nacional e a inovação nacional. Quanto as implicações empíricas deste estudo, os resultados obtidos poderão auxiliar a condução nacional de tomadores de decisões acerca de perspectivas culturais e incentivos à inovação, visando o crescimento e desenvolvimento econômico que agregue outros fatores importantes para uma nação tais como, produtividade, competitividade, tecnologias, empreendimentos e criatividade.

\section{REFERÊNCIAS}

AGUADO, R.; MARTINEZ, J. GDP and beyond: towards new measures of sustainability based on Catholic social thought. Asia-Pacific Journal of Business Administration, v. 4, n. 2, p. 124-138, 2012.

AGUIRRE-BASTOS, C.; WEBER, M. K. Foresight for shaping national innovation systems in developing economies. Technological Forecasting and Social Change, v.128, p. 186-196, 2017.

ANDREANO, M. S.; LAURETI, L.; POSTIGLIONE, P. Economic growth in MENA countries: Is there convergence of per-capita GDPs? Journal of Policy Modeling, v. 35, n. 4, p. 669-683, 2013.

ANGELIS, C. T. DE. The impact of national culture and knowledge management on governmental intelligence. Journal of Modelling in Management, v. 11, n. 1, p. 240-268, 2016.

BALZAT, M.; HANUSCH, H. Recent trends in the research on national innovation systems. Journal of Evolutionary Economics, v. 14, n. 2, p. 197-210, 2004.

BANCO MUNDIAL. $\quad$ GDP per capita. Disponibilidade: $<$ https://data.worldbank.org/indicator/NY.GDP.PCAP.CD >. [15 mai. 2018].

BANCO MUNDIAL. Who We Are. Disponibilidade: < http://www.worldbank.org/en/about/history >. [15 maio. 2018].

BANDARIN, F.; HOSAGRAHAR, J.; ALBERNAZ, F. S. Why development needs culture. Journal of Cultural Heritage Management and Sustainable Development, v. 1, n. 1, p. 15-25, 2011.

BANYA, R.M.; BIEKPE, N. Bank competition and economic growth: empirical evidence from selected frontier African countries. Journal of Economic Studies, Vol. 44 No. 2, p. 245-265, 2017.

CHEN, K; GUAN, J. Modeling the relative efficiency of national innovation systems. Research policy, v. 41 , n. 1, p. 102-115, 2012.

COUTO, J. P.; VIEIRA, J. C. National Culture and Research and Development Activities. Multinational Business Review, v. 12, n. 1, p. 19-36, 2004.

CRESWELL, J. W. Projeto de pesquisa: métodos qualitativo, quantitativo e misto. 5. ed. Porto Alegre: Artmed: Bookman, 2010.

DOSI, G. et al. Technical Change and Economic Theory. London: Pinter, 1988. 
EDQUIST, C. Systems of Innovation Technologies, Institutions and Organizations. London: Norton, 1997.

ESFAHANI, H.S. Exports, imports, and economic growth in semi-industrialized countries. Journal of Development Economics. Vol. 35 No. 1, pp. 93-116, 1991.

EVERDINGEN, Y. M. VAN; WAARTS, E. The Effect of National Culture on the Adoption of Innovations. Marketing Letters, v. 14, n. 3, p. 217-232, 2003.

FAGERBERG, J.; SAPPRASERT, K. National innovation systems: the emergence of a new approach. Science and public policy, v. 38, n. 9, p. 669-679, 2011.

FÁVERO, L. P. et al. Análise de dados: modelagem multivariada para a tomada de decisões. Rio de Janeiro: Campus, 2009.

FIELD, A. Descobrindo a estatística usando o SPSS. 2. ed. Porto Alegre: Artmed, 2009.

FREEMAN, C. Technology Policy and Economic Performance: Lessons from Japan. Pinter: London, 1987.

FREEMAN, C. Continental, national and sub-national innovation systems-complementarity and economic growth. Research policy, v. 31, n. 2, p. 191-211, 2002.

GII. In: Dutta, S., Lanvin, B., Wunsch-Vincent, S. (Eds.), The Global Innovation Index 2017: innovation feeding the world. Cornoll University, INSEAD, WIPO, 2017.

GODIN, B. National innovation system: The system approach in historical perspective. Science, Technology, \& Human Values, v. 34, n. 4, p. 476-501, 2009.

HAIR JR, J. F. et al. Fundamentos de métodos de pesquisa em administração. Porto Alegre: Bookman, 2005.

HOFSTEDE, G. Culture's consequences: international differences in work-related values. Beverly Hills, CA: Sage, 1980.

HOFSTEDE, G. Culture and Organizations. International Studies of Management \& Organization, v. 10, n. 4 , p. $15-41,1980$.

HOFSTEDE, G.; BOND, M. H. The Confucius connection: From cultural roots to economic growth. Organizational dynamics, v. 16, n. 4, p. 5-21, 1988.

HOFSTEDE, G.; MINKOV, M. Long-versus short-term orientation: new perspectives. Asia Pacific business review, v. 16, n. 4, p. 493-504, 2010.

HOFSTEDE, G. Dimensionalizing cultures: The Hofstede model in context. Online readings in psychology and culture, v. 2, n. 1, p. 8, 2011.

HOFSTEDE INSIGHTS. Country Comparison Tool. Disponibilidade: < https://www.hofstedeinsights.com/>. [15 maio 2018].

HOFSTEDE INSIGHTS. Who We Are. Disponibilidade: <https://www.hofstede-insights.com/aboutus/>. [09 jun. 2018].

IBGE. Produto Interno Bruto per capita. Disponibilidade: <https://seriesestatisticas.ibge.gov.br/series.aspx?vcodigo=SCN55>. [23 maio 2018].

IBGE. Produto Interno Bruto variação em volume taxa trimestral. Disponibilidade: $<$ https://seriesestatisticas.ibge.gov.br/series.aspx?vcodigo=ST12>. [23 maio 2018]. 
ÍNDICE DE INOVAÇÃO GLOBAL. Analysis: explore the interactive database of the Gll 2017 indicators. Disponibilidade: < https://www.globalinnovationindex.org/analysis-indicator >. [15 mai. 2018].

JOHNSON, B.; EDQUIST, C.; LUNDVALL, B. Economic development and the national system of innovation approach. First Globelics Conference, v. 1, p. 1-13, 2003.

KOSTIS, P. C.; KAFKA, K. I.; PETRAKIS, P. E. Cultural change and innovation performance. Journal of Business Research, v. 88, p. 306-313, 2018.

KRISTJÁNSDÓTTIR, H. et al. Hofstede national culture and international trade. Applied Economics, v. 49, n. 57, p. 5792-5801, 2017.

KWOK, C. C. Y.; TADESSE, S. National Culture and Financial Systems. Journal of International Business Studies, v. 37, n. 2, p. 227-247, 2006.

LASKOWSKA, I.; DAŃSKA-BORSIAK, B. The Importance of Human Capital For The Economic Development Of EU Regions. Comparative Economic Research, v. 19, n. 5, p. 63-79, 2016.

LU, W. M; KWEH, Q. L.; HUANG, C.L. Intellectual capital and national innovation systems performance. Knowledge-based systems, v. 71, p. 201-210, 2014.

LUNDVALL, B. National Systems of Innovation: Towards a Theory of Innovation and London: Interactive Learning. Pinter, 1992.

LUNDVALL, B. The Learning Economy and the Economics of Hope. New York: Anthem Press, 2016.

MARATTIN, L.; SALOTTI, S. Productivity and per capita GDP growth: The role of the forgotten factors. Economic Modelling, v. 28, n. 3, p. 1219-1225, 2011.

MAROCO, J. Análise estatística: com utilização do SPSS. Lisboa: Abril, 2003.

MONTAÑÉS, A.; OLMOS, L.; REYES, M. Has the Great Recession affected the convergence process? The case of Spanish provinces. Economic Modelling, v. 68, p. 360-371, 2018.

NELSON, R. R. National innovation systems: a retrospective on a study. Industrial and corporate change, v. 1, n. 2, p. 347-374, 1992.

NELSON, R.R. National Innovation Systems: A Comparative Analysis. Oxford University Press, 1993.

NELSON, R. R.; NELSON, K. Technology, institutions, and innovation systems. Research policy, v. 31, n. 2, p. 265-272, 2002.

OSTAPENKO, N. National culture, institutions and economic growth: The way of influence on productivity of entrepreneurship. Journal of Entrepreneurship and Public Policy, v. 4, n. 3, p. 331-351, 2015.

PATEL, P.; PAVITT, K. National innovation systems: why they are important, and how they might be measured and compared. Economics of innovation and new technology, v. 3, n. 1, p. 77-95, 1994.

PATTILLO, C., POIRSON, H.; RICCI, L. What are the channels through which external debt affects growth? IMF Working Paper, 2004.

PRADHAN, R. P.; ARVIN, M. B.; BAHMANI, S. Are innovation and financial development causative factors in economic growth? Evidence from a panel granger causality test. Technological Forecasting and Social Change, v. 132, p.130-142, 2018.

RAUCH, A. et al. National culture and cultural orientations of owners affecting the innovation-growth relationship in five countries. Entrepreneurship \& Regional Development, v. 25, n. 9-10, p. 732-755, 2013. 
ROMER, P.M. Endogenous technological change. Journal of Political Economy, Vol. 98 No. 5, p. 71 S102, 1990.

SHANE, S. Cultural influences on national rates of innovation. Journal of Business Venturing, v. 8, $\mathrm{n}$. 1, p. 59-73, 1993.

SHANE, S.; VENKATARAMAN, S.; MACMILLAN, I. Cultural Differences in Innovation Championing Strategies. Journal of Management, v. 21, n. 5, p. 931-952, 1995.

SHARIPOV, I. Exogenous vs Endogenous Growth in the EU's EaP and Central Asian Countries. Scientific Annals of Economics and Business, v. 63, n. s1, p. 109-124, 2016.

SU, Y.; LIU, Z. The impact of foreign direct investment and human capital on economic growth: Evidence from Chinese cities. China Economic Review, v. 37, p. 97-109, 2016.

WAARTS, E.; EVERDINGEN, Y. VAN. The Influence of National Culture on the Adoption Status of Innovations: An Empirical Study of Firms Across Europe. European Management Journal, v. 23, n. 6, p. 601-610, 2005.

ZHAO, X.; LI, H.; RAUCH, A. Cross-country differences in entrepreneurial activity: The role of cultural practice and national wealth. Frontiers of Business Research in China, v. 6, n. 4, p. 447-474, 2012. 